Urologe $2021 \cdot 60$ (Suppl 1):S1-S2

https://doi.org/10.1007/s00120-021-01625-2

Angenommen: 28. Juli 2021

(c) Springer Medizin Verlag GmbH, ein Teil von Springer Nature 2021

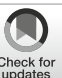

\title{
Zum 73. Kongress der Deutschen Gesellschaft für Urologie
}

\author{
eUrologie
}

\author{
Arnulf Stenzl \\ Klinik für Urologie, Universitätsklinikum Tübingen, Tübingen, Deutschland
}

Liebe Kolleginnen und Kollegen, sehr geehrte Damen und Herren,

ich freue mich Sie am 15.09.2021 zum 73. Kongress der Deutschen Gesellschaft für Urologie - nach dem virtuellen Zwischenspiel 2020 - begrüßen zu dürfen. Die Mitglieder der Programmkommission, die Vorsitzenden und Mitglieder der Arbeitskreise und alle Gutachter und Obergutachter haben auch in diesem Jahr mit vielen virtuellen Sitzungen ein spannendes und aktuelles Programm zusammengestellt. Zum Unterschied vom letzten Jahr konnte allerdings keine einzige Programmkommission in physischer Anwesenheit der Beteiligten stattfinden. Aber so machten wir es in diesem Jahr umgekehrt: Programmsitzungen virtuell aber den Kongress als Präsenzveranstaltung.

In diesem Jahr findet der offizielle Beginn des Kongresses bereits am Mittwoch dem 15. September 2021 mit einer Plenarveranstaltung, gestaltet vom Vorstand der Deutschen Gesellschaft für Urologie (DGU), statt. Des Weiteren sind die Plenarsitzungen am Donnerstag und Freitag als „Standalone-Sitzungen" zwischen vormittags geplant, die mit namhaften, über die Grenzen Deutschlands hinaus bekannten Rednern zum Thema Digitalisierung in der Urologischen Praxis und Klinik und neuen Ansätzen in der Methodik der Behandlung des Prostatakarzinoms besetzt sein werden.

Neu ist auch, dass durch die Einführung von verkürzten freien Beiträgen ( $3 \mathrm{~K}$, kurz - kreativ - knackig) die Anzahl der Parallelveranstaltungen reduziert werden konnte. Diese 3K-Beiträge können durch Abruf auf der Homepage der DGU vertieft werden und sind so nachhaltiger. Alle Bereiche der Urologie - von der Uroonko- logie über funktionelle Urologie, Kinderurologie, Andrologie, Transplantation, Neurourologie und weitere Subspezialitäten sind mit spannenden Beiträgen vertreten. Dem Wunsch vieler Teilnehmer entsprechend werden viele Diskussionen, Rundtischgespräche mit neuen Formaten, die die Diskussionen lebendiger machen, auf dem Kongress zu finden sein.

Während des ganzen Kongresszeitraums wird es durchgehend Filme (,semilive") zu den verschiedensten Operationstechniken in der Urologie geben.

Was kann man denn eigentlich aus diesem Präsenzkongress mitnehmen? Neben Live-Vorträgen und Diskussionen sowie den oben beschriebenen 3K-Beiträgen werden ausgesuchte Mitschnitte auch noch nach dem Kongress zur weiteren Vertiefung abrufbar sein. Eine Hilfe wird das Abschlussplenum am Samstag sein: Hier werden die "Souvenirs" des diesjährigen Kongresses präsentiert zusammen mit den möglichen Auswirkungen auf die Urologie in den Jahren nach dem Kongress. Freuen Sie sich mit uns auf einen spannenden aber auch unterhaltsamen und tiefgründigen Präsenzkongress in einem von der Anbindung her in Deutschland einzigartigem und mit modernsten Einrichtungen ausgestatteten Kongresszentrum.

Das Team der Urologie Tübingen, die Kongressveranstalter, Programmkommission aber auch die Sponsoren freuen sich auf Ihr Kommen.

Prof. Dr. med. Dr. h.c. Arnulf Stenzl Ärztlicher Direktor Universitätsklinikum Tübingen Klinik für Urologie Präsident der Deutschen Gesellschaft für Urologie 


\section{Editorial}

\section{Korrespondenzadresse}

Prof. Dr. med. Dr. h.c. Arnulf Stenzl Hoppe-Seyler-Straße 3, 72076 Tübingen,

Deutschland

Arnulf.Stenzl@med.uni-tuebingen.de

Interessenkonflikt. Prof. A. Stenzl ist Präsident der Deutschen Gesellschaft für Urologie 2020/2021. 